\title{
Flat topped hypopigmented micropapules on the flank and neck
}

\section{Ania Henning, Joshua Weaver}

Department of Pathology and Laboratory Medicine, Summa Health System-Akron City, 525 E Market St, Akron, OH 44304, USA

Corresponding author: Ania Henning, M.D, E-mail: aniahenning@gmail.com

\begin{abstract}
Lichen nitidus (LN) is a rare inflammatory condition which presents as multiple small, shiny, pale to flesh colored papules measuring 1-2mm in diameter. Lesions typically occur on the abdomen, chest, genitalia and extremities. However, cases of LN involving the nails, oral mucosa and acral skin have been described. Although, it typically presents in children and young adults, there is no gender or racial preference. Clinical and histopathological findings are characteristic for LN and treatment is rarely warranted. Herein we report a case of LN in a 26-year-old African American female who presented with multiple areas of $\mathrm{LN}$.
\end{abstract}

Key words: Lichen nitidus; Rash; Lichen planus; Papules

\section{INTRODUCTION}

$(\mathrm{LN})$ is a rare condition with poorly understood pathophysiology [1,2]. Patients are usually asymptomatic but may have pruritis. Clinical presentation may be sufficient enough for diagnosis. However, patients with symptoms may warrant a biopsy, especially if seen by generalized practitioners. It is most common in children and young adults; however, it has also been described in older patients $[3,4]$. Both genders and all races may be affected. Many clinical variants of LN which have been described in literature including vesicular, confluent, generalized, perforating, follicular and linear forms $[1,4]$. Although $\mathrm{LN}$ is generally selflimited, clinical presentation and biopsy results may be needed for patient reassurance.

\section{CASE REPORT}

A 26-year-old pregnant African American female presented with complaints of a rash present on her right flank and the right side of her neck (Figs. 1 and 2). The rash was chronic in nature and present for approximately 2 years. Patient reported minor pruritis at times but denied dry, cracked, scaling skin, or any changes to the rash. She was evaluated for the rash previously at an outpatient clinic and was prescribed an antifungal cream for tinea. She used the antifungal cream for 3 months without any improvement. Clinically the rash was discrete with multiple pinpoint, flat-topped, pink colored papules. A shave biopsy was taken from the papules present on the flank and sent to pathology.

Sections of the biopsy revealed a patchy / multifocal lymphohistiocytic infiltrate obscuring the epidermaldermal junction and enclosed within an epidermal collaret (Fig. 3). Subtle basal cell vacuolization and very rare apoptotic keratinocytes were noted in these areas. Overlying thinning of the epidermis was noted (Fig. 4). Eosinophils were not identified. The overlying granular cell layer, and stratum corneum with otherwise fairly unremarkable. These morphologic features of multiple small areas of a more mixed histiocytic and lymphocytic infiltrate surrounded by epidermal collarets was diagnostic for lichen nitidus.

The patient was informed of her biopsy results. However, she was pregnant and therefore, treatment

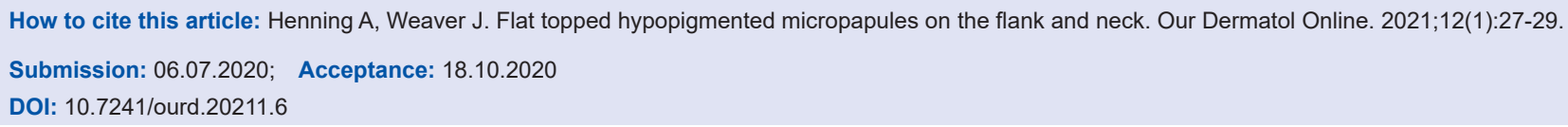




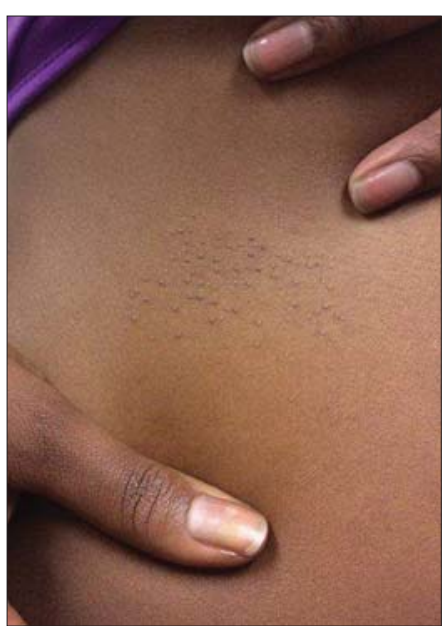

Figure 1: Discrete small flesh colored micropapules on the flank.

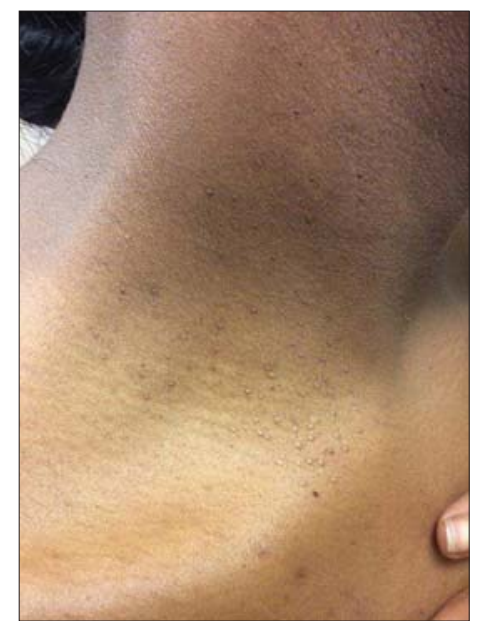

Figure 2: Shiny slightly hypopigmented micropapules on the neck.

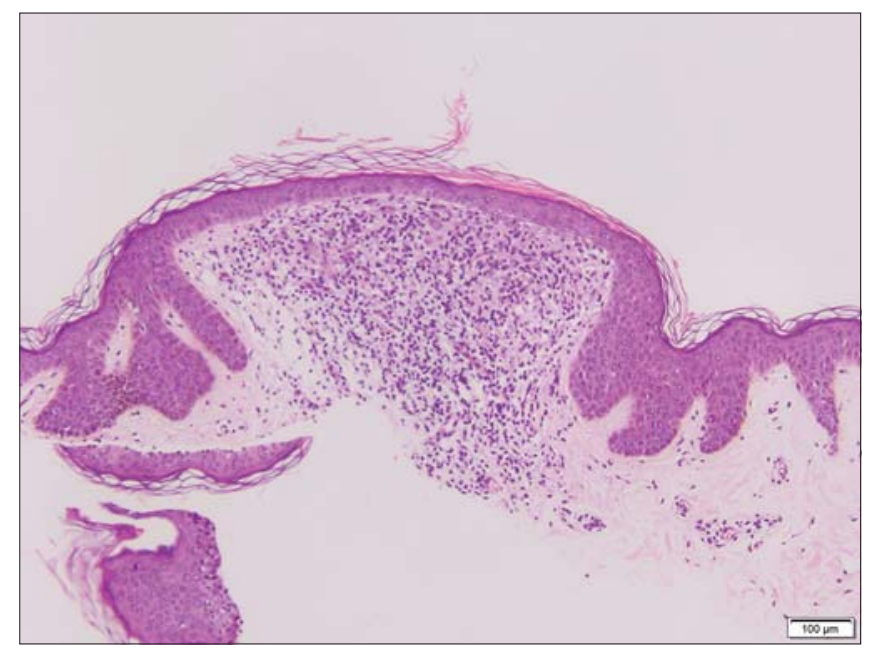

Figure 3: Multifocal lymphohistiocytic infiltrate obscuring the epidermaldermal junction and enclosed within an epidermal collaret. H\&E 100x.

was not recommended unless the rash became worse or more problematic. She was reassured and instructed to schedule a follow-up if the rash progressed.

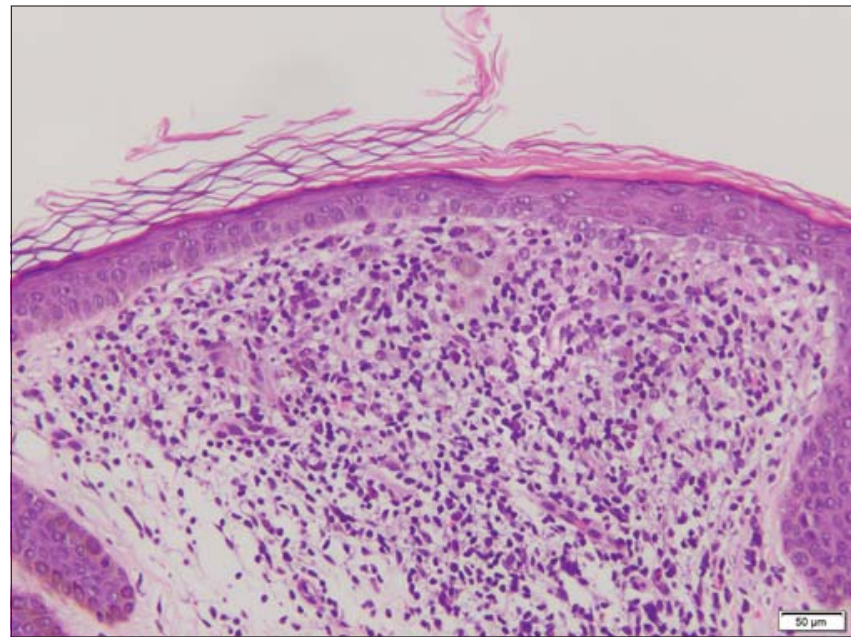

Figure 4: Mixed histiocytic and lymphocytic infiltrate, an overlying thinning epidermis, and scattered apoptotic keratinocytes. H\&E 200x.

\section{DISCUSSION}

Histopathology of $\mathrm{LN}$ is very characteristic and consistent with a well circumscribed lymphohistiocytic infiltrate hugging the epidermis. The overlying epidermis is typically atrophic and parakeratotic. The rete ridges extend downward, making finger like projections enclosing the inflammatory infiltrate in a typical ball in claw appearance. [5] Basal cell hydropic degeneration and cytoid bodies may be seen $[1,5]$.

Older lesions of LN may have granulomas and giant cells while earlier lesions typically consist of lymphocytes [5]. Immunohistochemical stains (IHC), although unnecessary can also be utilized. LN demonstrates a heterogenous population of CD4+ and CD8+ lymphocytes along with macrophages [6].

The differential diagnosis of LN includes lichen planus (LP) and in fact many authors have described $\mathrm{LN}$ and LP occurring in the same patient population. This has sparked the debate as to whether $\mathrm{LN}$ is merely a micropapular variant of LP [6]. However, both entities have distinct histopathological and IHC patterns. LP, in contrast to $\mathrm{LN}$ demonstrates a predominance of CD $4+$ helper T-cells along with HECA-452 staining as well as a more uniform lymphocytic infiltrate $[1,6]$.

Although histology and clinical presentation are characteristic; features of dermoscopic findings are notable and thus, dermoscopy may provide to be a useful tool [1]. Jahkar et al, studied 8 cases of LN using polarized and non-polarized dermoscopy. Nonpolarizing dermoscopy was characteristic for shiny 
elevated surface with the absence of dermatoglyphics as well as central depression. Polarizing dermoscopy showed the presence of ill-defined hypopigmentation, diffuse erythema as well as linear vessels [5]. Features of dermoscopic findings correlated with the histopathologic findings of LN. The absence of dermatoglyphics correlated with the flattening features of the epidermis overlying the inflammatory infiltrate and the central depression seen was characteristic for the well circumscribed ball like inflammatory infiltrate [5]. Therefore, dermoscopy can be used as a non-invasive diagnostic tool to help differentiate between other cutaneous lesions.

The pathogenesis of $\mathrm{LN}$ is unclear. It has been linked to immunologic alterations in patients $[1,6]$. Reports of LN associated with cutaneous disease such as lichen planus and erythema nodosum have been described, suggesting that an allergen may cause antigen presenting cells to activate a cell mediated response which results in the characteristic lymphocytic accumulation [1]. Furthermore, LN has been associated with a variety of systemic conditions including Niemen Pick's disease, HIV, Chron's disease and Down's Syndrome $[1,6]$. Generalized LN, on the other hand, has been reported following treatment using interferon alpha, ribavirin and anti PD-1 therapy such as nivolumab $[1,7]$.

$\mathrm{LN}$ is generally a self-limiting eruption, however the clinical course is uncertain [8]. Patients typically have spontaneous resolution of symptoms and thus treatment is often not necessary given the asymptomatic nature [6]. However, patients who present with symptoms or have cosmetically disrupting lesions may be treated. Symptomatic patients or patients with chronic LN may benefit from topical or systemic corticosteroids $[1,6]$. Patients with the generalized form can be treated with PUVA or astemizole. Consequently, oral retinoids have proved to be beneficial in patients who have involvement of acral skin [1].

\section{CONCLUSION}

Most lesions of $\mathrm{LN}$ resolve within $1-3$ years of onset $[6,9]$. However, the papules of LN may lead to post inflammatory hyperpigmentation. It is believed that as the papules heal, they are replaced by hyperpigmented post inflammatory macules which tend to resolve within months $[1,9]$. Treatment may be necessary for cosmetic purpose as well. It is important for practioners to be aware of LN. Our patient was originally misdiagnosed and treated for tinea unnecessarily. Fortunately, since biopsy and reassurance she has not had any further complaints.

\section{Consent}

The examination of the patient was conducted according to the principles of the Declaration of Helsinki.

The authors certify that they have obtained all appropriate patient consent forms, in which the patients gave their consent for images and other clinical information to be included in the journal. The patients understand that their names and initials will not be published and due effort will be made to conceal their identity, but that anonymity cannot be guaranteed.

\section{REFERENCES}

1. Schwartz C, Goodman MB. Lichen Nitidus. [Updated 2019 Dec 3]. In: StatPearls [Internet]. Treasure Island (FL): StatPearls Publishing; 2020 Jan-. Available from: https://www.ncbi.nlm.nih.gov/books/ NBK551709/

2. Cho EB, Kim HY, Park EJ, Kwon IH, Kim KH, Kim KJ. Three cases of lichen nitidus associated with various cutaneous diseases. Ann Dermatol. 2014;26:505-9.

3. Chen W, Schramm M, Zouboulis CC. Generalized lichen nitidus. J Am Acad Dermatol. 1997;36:630-1.

4. Kundak S, Çakır Y. Pediatric lichen nitidus: A single-center experience. Pediatr Dermatol. 2019;36:189-92.

5. Jakhar D, Grover C, Kaur I, Sharma S. Dermatoscopic features of lichen nitidus. Pediatr Dermatol. 2018;35:866-7.

6. Payette MJ, Weston G, Humphrey S, Yu J, Holland KE. Lichen planus and other lichenoid dermatoses: Kids are not just little people. Clin Dermatol. 2015;33:631-43.

7. Cho M, Nonomura Y, Kaku Y, Dainichi T, Otsuka A, Kabashima K. Generalized lichen nitidus following anti-PD-1 antibody treatment. JAMA Dermatol. 2018;154:367-9.

8. Rallis E, Verros C, Moussatou V, Sambaziotis D, Papadakis P. Generalized purpuric lichen nitidus. Report of a case and review of the literature. Dermatol Online J. 2007;13:5.

9. Chu J, Lam JM. Lichen nitidus. CMAJ. 2014;186:E688.

Copyright by Ania Henning, et al. This is an open-access article distributed under the terms of the Creative Commons Attribution License, which permits unrestricted use, distribution, and reproduction in any medium, provided the original author and source are credited.

Source of Support: Nil, Conflict of Interest: None declared. 